\title{
Lifetime effects, costs, and cost effectiveness of testing for human papillomavirus to manage low grade cytological abnormalities: results of the NHS pilot studies
}

Rosa Legood, Alastair Gray, Jane Wolstenholme, Sue Moss

\begin{abstract}
Objectives To predict the incremental lifetime effects, costs, and cost effectiveness of using human papillomavirus testing to triage women with borderline or mildly dyskaryotic cervical smear results for immediate colposcopy.

Design Modelling study.

Setting Three centres participating in NHS pilot studies, United Kingdom.

Population Women aged 25-64 with borderline or mildly dyskaryotic cervical smear results.

Interventions Screening using conventional cytology, liquid based cytology, and four strategies with different age cut-off points and follow up times that used combined liquid based cytology and human papillomavirus testing (adjunctive human papillomavirus testing).

Results The model predicts that compared with using conventional cytology without testing for human papillomavirus, testing for the virus in conjunction with liquid based cytology for women with borderline or mildly dyskaryotic cervical smear results (aged 35 or more) would cost $£ 3735$ (euros5528; \$6474) per life year saved. Extending adjunctive human papillomavirus testing in combination with liquid based cytology to include women aged between 25 and 34 costs an additional $£ 4233$ per life year saved. Human papillomavirus testing is likely to reduce lifetime repeat smears by $52 \%-86 \%$ but increase lifetime colposcopies by $64 \%-138 \%$.

Conclusions Testing for human papillomavirus to manage all women with borderline or mildly dyskaryotic cervical smear results is likely to be cost effective. The predicted increase in lifetime colposcopies, however, deserves careful consideration.
\end{abstract}

\section{Introduction}

Human papillomavirus is present in virtually all cases of cervical cancer. ${ }^{1}$ Testing for the virus could be incorporated in a cervical cancer screening programme to stratify women with minor cytological abnormalities for immediate colposcopy. ${ }^{23}$ In the United Kingdom, women with borderline or mildly dyskaryotic cervical smear results are recalled for repeat smears every six months and only return to routine screening intervals after three consecutive negative test results. The disadvantages of this approach compared with testing for human papillomavirus are potentially missing lesions that could be treated earlier, the costs incurred by patients and the healthcare system, and the failure of women to attend for repeat tests.
In 2000-1 the Department of Health established a series of pilot sites to assess both liquid based cytology and human papillomavirus testing of women with borderline or mildly dyskaryotic smear results. Three laboratories were selected after a competitive process and converted to using liquid based cytology to prioritise women for immediate referral for colposcopy. The Department of Health policy research programme commissioned an independent evaluation of these pilots. The clinical and epidemiological outcomes observed at 12 months are presented elsewhere. ${ }^{4}$

We compared the lifetime effects, costs, and cost effectiveness of using cytology alone with using combined cytology and triage on the basis of human papillomavirus testing to manage women with borderline and mildly dyskaryotic smear results in the United Kingdom. We used the current policy of screening women aged 25-49 every three years and women aged 50-64 every five years.

\section{Methods}

We used the final results of the NHS pilot studies, including clinical, epidemiological, and cost data. As no long term follow-up data are available, we used a mathematical model to estimate the lifetime effects, costs, and cost effectiveness. We used an adapted version of a prior natural history model developed using a Markov process in Data 4.0 (Treeage software; Williamstown, MA). ${ }^{5}$ Our model predicted the lifetime costs and effects of alternative strategies for screening from age 15 to death. The analysis was from the health service perspective.

\section{Screening strategies}

We compared current screening protocols using conventional cytology with five alternative strategies (box). In all strategies, women with moderate or severe cytology results are referred directly for colposcopy; inadequate cytology results are retested (this is assumed to occur immediately); and women with normal results return to routine screening.

When only cytology was used for repeat testing every six months (strategies $\mathrm{A}$ and $\mathrm{E}$ and women aged less than 35 in strategy D), women were referred for colposcopy after three borderline or two mildly dyskaryotic smear results. Women only returned to routine screening after three consecutive negative results, again at six month intervals.

People involved in study, a table, and figure are on bmj.com 


\section{Research}

When cytology and human papillomavirus tests were used for repeat testing, women were referred for colposcopy if the repeat test was positive for human papillomavirus, or the cytology result was mild dyskaryosis or worse, or both, otherwise they returned to routine screening.

\section{Natural history}

From the literature we took the probability of transitions between health states (healthy, human papillomavirus only, cervical intraepithelial neoplasia (CIN) grade 1, CIN-2, or CIN-3 and invasive cancer stages I-IV), and the probability of symptoms in an unscreened population. ${ }^{5}$ We chose the natural history model as it has been validated ${ }^{5}$ and reflects current scientific understanding of preinvasive disease. Figure 1 illustrates the health states defined by the model and the potential transitions between states.

All probabilities of transition were calculated for a six month time frame, reflecting the cycle length of the model. We adapted the model for the United Kingdom using local data on survival from invasive cancer ${ }^{6}$ and mortality from other causes. ${ }^{78}$ Table 1 shows these and other variables in the model.

We assumed that all cases of preinvasive and invasive cervical cancer begin with human papillomavirus infection, that the American categories for low grade and high grade squamous intraepithelial lesions are equivalent to CIN-1 and CIN-2 or CIN-3, and that women who survive after five years have the same life expectancy as women in the general population. ${ }^{15}$

\section{Attendance}

Attendance rates at routine screening were based on the percentage of eligible women who attended at least once over a five year period. ${ }^{10}$ We estimated attendance rates for repeat screening and colposcopy using data from the pilots. ${ }^{4}$ We

\section{Alternative strategies for screening women for cervical cancer \\ Strategy A \\ Liquid based cytology only \\ Strategy B \\ Combined liquid based cytology and human papillomavirus testing (hybrid capture II assay for human papillomavirus DNA; Digene, Abbott, Maidenhead) for women with borderline or mildly dyskaryotic results. Women who test positive for the virus are referred for immediate colposcopy and women who test negative are recalled at six months for repeat cytology and human papillomavirus testing (this strategy reflects the original pilot protocol) \\ Strategy C \\ Same as strategy B except that women aged less than 35 who initially tested positive are not referred for colposcopy. Even if repeat tests for these women give negative results, they are recalled for a third combined test at 12 months (this strategy reflects the amended pilot protocol) \\ Strategy D \\ Combined liquid based cytology and adjunctive human papillomavirus testing (as described in strategy B) for women aged 35 or more, and liquid based cytology only for women under 35 (as described in strategy A) \\ Strategy E \\ Combined liquid based cytology and adjunctive human papillomavirus testing (as described in strategy B) but no testing for human papillomavirus in repeat tests}

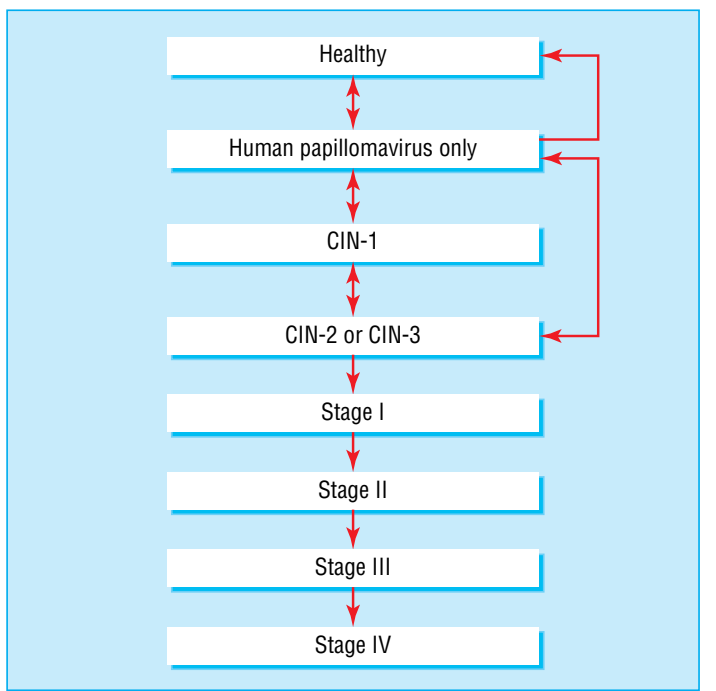

Fig 1 Natural history of disease model. Death from cervical cancer or from other causes not shown. CIN=cervical intraepithelial neoplasia

assumed that if women did not attend they would only be recalled for screening at the next screening round.

\section{Effectiveness of screening}

Estimates of sensitivity and specificity for human papillomavirus using the hybrid capture II assay were identified from a meta-analysis. ${ }^{11}$ These studies followed-up women with both positive and negative test results at colposcopy or histology thus minimising verification bias. It is assumed that sensitivity and specificity for human papillomavirus was the same for CIN-1 and only human papillomavirus.

At baseline we used estimates of the sensitivity and specificity of conventional cytology from an earlier study. ${ }^{16}$ A recent metaanalysis emphasised the failure of some trials to meet several validity criteria but indicated that overall liquid based cytology shows an $11 \% \quad(95 \%$ confidence interval $3 \%$ to $20 \%)$ improvement in sensitivity to detect CIN- $1 .{ }^{17}$ Using the positive predictive values obtained in the pilots we have estimated an improvement in the sensitivity of CIN-2 or CIN-3 ranging from $3.6 \%(95 \%$ confidence interval $-1.2 \%$ to $5.0 \%)$.

Little data exist on the sensitivity of cytology testing and human papillomavirus testing strategies for the detection of invasive cancer. We have not modelled in full the screening strategies for invasive cancer, and we have assumed that for all strategies $90 \%$ of cases of invasive cancer are detected at each screening round. All colposcopies were assumed to be 100\% sensitive and specific, and we assumed that all abnormalities when found at colposcopy are treated. It was assumed that colposcopic treatment is $90 \%$ effective (range $80 \%-100 \%),{ }^{12}$ and that $90 \%$ (range $0 \%$ to $100 \%$ ) of women return to a healthy state with no human papillomavirus infection. ${ }^{5}$

\section{Costs}

We used data from the pilot sites to calculate the unit costs of liquid based cytology, conventional cytology, and human papillomavirus testing. Staff time was estimated from record sheets sent to a random sample of smear takers and all smear readers at the laboratories. We obtained costs of conventional cytology equipment and consumables from the laboratory; estimates of the indicative market price for liquid based cytology and human papillomavirus equipment were made in consultation with the NHS Purchasing and Supplies Agency. Unit costs for primary care were taken from the literature and laboratory staff costs 
Research

Table 1 Summary of parameters in cost effectiveness model

\begin{tabular}{|c|c|c|c|c|c|}
\hline Parameters & Baseline & Minimum & Maximum & Distribution & Reference \\
\hline \multicolumn{6}{|l|}{ Management variables } \\
\hline \multicolumn{6}{|l|}{ Yearly (\%) discount rate*: } \\
\hline Costs & 3.5 & 0 & 6 & - & 9 \\
\hline Effects & 3.5 & 0 & 6 & - & 9 \\
\hline \multicolumn{6}{|l|}{ Age: } \\
\hline Start of screening & 25 & & & - & \\
\hline End of screening & 64 & & & - & \\
\hline \multicolumn{6}{|l|}{ Screening interval: } \\
\hline Age 25 to 49 & 3 yearly & & & - & \\
\hline Age 50 to 64 & 5 yearly & & & - & \\
\hline \multicolumn{6}{|l|}{ Attendance (\%): } \\
\hline Routine smear & 81 & 76 & 85 & $\beta$ & 10 \\
\hline Repeat smear (age <35) & 79 & 77 & 80 & $\beta$ & 4 \\
\hline Repeat smear (age $\geq 35$ ) & 85 & 84 & 87 & $\beta$ & 4 \\
\hline Colposcopy (age <35) & 95 & 97 & 93 & $\beta$ & 4 \\
\hline Colposcopy (age $\geq 35$ ) & 93 & 90 & 96 & $\beta$ & 4 \\
\hline \multicolumn{6}{|c|}{ Transition probabilities and incidence rates of preinvasive disease (6 months) } \\
\hline Prevalence of human papillomavirus infection age $15 \dagger$ & 0.1 & & & & 5 \\
\hline Prevalence of $\mathrm{CIN}-1$ age $15 \dagger$ & 0.01 & & & & 5 \\
\hline Age specific incidence of human papillomavirus infection: & & & & & 5 \\
\hline 15 to 19 & $0.051-0.089$ & & & & 5 \\
\hline 20 to 23 & $0.078-0.051$ & & & & 5 \\
\hline 24 to 29 & 0.025 & & & & 5 \\
\hline 30 to 49 & 0.005 & & & & 5 \\
\hline$\geq 50$ & 0.003 & & & & 5 \\
\hline \multicolumn{6}{|l|}{ Age specific regression rate, human papillomavirus infection: } \\
\hline 15 to 24 & 0.33 & 0.26 & 0.54 & $\beta$ & 5 \\
\hline 25 to 29 & 0.21 & 0.18 & 0.26 & $\beta$ & 5 \\
\hline$\geq 30$ & 0.05 & 0.03 & 0.07 & $\beta$ & 5 \\
\hline Progression rate (human papillomavirus to $\mathrm{CIN}-1$ ) & 0.04 & 0.03 & 0.06 & $\beta$ & 5 \\
\hline $\begin{array}{l}\text { Proportion of human papillomavirus infections progressing to CIN-2 or } \\
\text { CIN-3 }\end{array}$ & 0.1 & 0.05 & 0.5 & $\beta$ & 5 \\
\hline \multicolumn{6}{|l|}{ Regression rate CIN-1 to human papillomavirus or healthy: } \\
\hline Age 15 to 34 & 0.084 & 0.074 & 0.126 & $\beta$ & 5 \\
\hline Age $\geq 35$ & 0.042 & 0.029 & 0.074 & $\beta$ & 5 \\
\hline Proportion of CIN-1 reverting to healthy & 0.9 & 0.5 & 1.0 & $\beta$ & 5 \\
\hline \multicolumn{6}{|l|}{ Progression rate $\mathrm{CIN}-1$ to $\mathrm{CIN}-2$ or $\mathrm{CIN}-3$} \\
\hline Age 15 to 34 & 0.0087 & 0.0087 & 0.029 & $\beta$ & 5 \\
\hline Age $\geq 35$ & 0.035 & 0.029 & 0.056 & $\beta$ & 5 \\
\hline Regression rate $\mathrm{CIN} 2 / 3$ to CIN1 or healthy & 0.035 & 0.0292 & 0.056 & $\beta$ & 5 \\
\hline Proportion of CIN2/3 reverting to healthy & 0.5 & 0 & 0.5 & $\beta$ & 5 \\
\hline Progression rate CIN2/3 to invasive cancer & 0.025 & 0.018 & 0.034 & $\beta$ & 5 \\
\hline \multicolumn{6}{|l|}{ Effectiveness of screening } \\
\hline \multicolumn{6}{|l|}{ Human papillomavirus testing: } \\
\hline Sensitivity & 0.948 & 0.927 & 0.969 & Uniform & 11 \\
\hline Specificity & 0.673 & 0.582 & 0.764 & Uniform & 11 \\
\hline Effectiveness (\%) of colposcopy & 90 & 80 & 100 & Uniform & 12 \\
\hline Progression rate stage I to stage II & 0.13 & - & - & - & 5 \\
\hline Probability of symptoms stage I & 0.08 & - & - & - & 5 \\
\hline Progression rate stage II to stage III & 0.23 & - & - & - & 5 \\
\hline Probability of symptoms stage II & 0.12 & - & - & - & 5 \\
\hline Progression rate stage III to stage IV & 0.44 & - & - & - & 5 \\
\hline Probability of symptoms stage III & 0.37 & - & - & - & 5 \\
\hline Probability of symptoms stage IV & 0.68 & - & - & - & 5 \\
\hline \multicolumn{6}{|l|}{ Annual probability of survival after invasive cancer diagnosisł } \\
\hline Stage I & $0.97-0.99$ & & & $\beta$ & 8 \\
\hline Stage II & $0.77-0.96$ & & & $\beta$ & 8 \\
\hline Stage III & $0.54-0.90$ & & & $\beta$ & 8 \\
\hline Stage IV & $0.49-0.88$ & & & $\beta$ & 8 \\
\hline \multicolumn{6}{|l|}{ Costs (2001-2 prices) } \\
\hline Conventional cytology $\dagger$ & 23.6 & 23.4 & 23.8 & $\gamma$ & \\
\hline Liquid based cytology $\dagger$ & 25.7 & 23.5 & 28.2 & $\gamma$ & \\
\hline Human papillomavirus test & 20.5 & 10.3 & 34.5 & $\gamma$ & \\
\hline Colposcopy outpatients (no CIN) & 122 & 98 & 147 & $\gamma$ & 13 \\
\hline Colposcopy and treatment for CIN & 624 & 415 & 833 & $\gamma$ & 14 \\
\hline
\end{tabular}




\begin{tabular}{|c|c|c|c|c|c|}
\hline Parameters & Baseline & Minimum & Maximum & Distribution & Reference \\
\hline \multicolumn{6}{|c|}{5 year treatment costs $(£)$ after detection: } \\
\hline Stage I & 10702 & 9354 & 12041 & $\gamma$ & 14 \\
\hline Stage II & 17629 & 14279 & 20982 & $\gamma$ & 14 \\
\hline Stage III & 17513 & 13880 & 21143 & $\gamma$ & 14 \\
\hline Stage IV & 18639 & 8147 & 29129 & $\gamma$ & 14 \\
\hline
\end{tabular}

$\mathrm{CIN}=$ cervical intraepithelial neoplasia.

* $3 \%$ after 30 years.

†Age specific.

†Estimates vary by year since diagnosis.

§Includes adjustment for inadequate rates.

estimated using the mid-point of staff salaries. ${ }^{18}$ We adjusted the costs for cytology to incorporate the cytology results that were inadequate (that is, where slides were not able to be interpreted for technical reasons). ${ }^{4}$ It was assumed that kits to test for human papillomavirus are used to process a batch of samples at full capacity. All costs are converted to 2001-2 prices using the NHS Health and Community Price Index and are reported in sterling.

We used a detailed patient audit of the costs of invasive cancer over five years, including treatment and palliative care. ${ }^{14} \mathrm{We}$ assumed that no additional costs would be incurred beyond this period. ${ }^{15}$ We also used audit data on costs associated with cervical intraepithelial neoplasia, including initial diagnosis at colposcopy, management (for example, loop cone biopsy), and any subsequent colposcopy follow-up (including adverse events). ${ }^{14} \mathrm{~A}$ single outpatient attendance was used as a proxy cost for a colposcopy when there was no cervical intraepithelial neoplasia. $^{13}$

\section{Analysis}

Following current UK recommendations, we discounted future costs and future benefits at 3.5\% for the first 30 years and 3\% thereafter. ${ }^{9}$ To estimate the comparative cost effectiveness between the strategies, we first ranked the strategies in ascending order of effectiveness. We excluded options that were dominated (that is, less effective and more costly than an alternative) and strategies that were extended dominated (that is, inside the cost effectiveness frontier). For the remaining strategies we calculated the incremental costs, effects, and resulting cost effectiveness ratios (costs divided by effects).

To test the effect of uncertainty about a variable, we carried out one way and probabilistic sensitivity analyses. In the one way sensitivity analysis we used the minimum and maximum estimates for each variable. As the data for estimating the sensitivity of conventional cytology were based on one UK study, we used additional data from the international literature to test uncertainty around these estimates. We carried out a random effects meta-analysis to synthesise the result of a systematic review. ${ }^{19}$ Nine studies were identified that did not have verification bias and were carried out in routine low risk settings. The combined estimate of sensitivity to detect CIN-1 was 0.46
(95\% confidence interval 0.29 to 0.72 ) and to detect CIN-2 or CIN-3 was 0.84 (0.75 to 0.94 ).

We also undertook a probabilistic sensitivity analysis. ${ }^{20}$ Appropriate distributions were assigned depending on the availability of data and type of variable (see table 1). Using simulation, we generated a series of estimates for the costs and effects of each strategy by sampling from the distribution of each model variable. We then used a net benefit framework to plot cost effectiveness acceptability curves showing optimal strategies at different values that society would be willing to pay for a gain in life years (see bmj.com). ${ }^{21}$

\section{Results}

Our model predicted a peak prevalence for human papillomavirus at 22 years of age, tailing off significantly after age 30 . The prevalence for CIN-1 peaks at age 25. The rates of human papillomavirus for women aged 30-60 are consistent with UK data on human papillomavirus prevalence collected in the HPV in addition to routine testing trial. ${ }^{22}$ Allowing for death from other causes, the model predicts a lifetime risk of death from invasive cancer of $1.4 \%$. This estimate is consistent with modelling estimates from a recent study, which predicted that in the absence of screening the lifetime risk of death from invasive cancer was $1.5 \%$ for women born after $1950 .^{23}$

Table 2 reports the baseline point estimates for the cost effectiveness results, which are illustrated in figure 2. Compared with screening using conventional cytology, the next most cost effective strategy seems to be combined liquid based cytology and human papillomavirus testing to prioritise women aged 35 or more with borderline or mildly dyskaryotic smear results for immediate colposcopy (strategy D). Although liquid based cytology alone (strategy A) is cheaper than strategy $\mathrm{D}$, it also seems less effective and has a higher cost effectiveness ratio. Therefore it is likely to be more cost effective to use strategy D.

Strategies B, C, and E (human papillomavirus testing used to triage women of all ages with borderline or mild results) provide additional health gain compared with strategy D (human papillomavirus testing restricted to only women aged more than 35)

Table 2 Baseline estimates of lifetime discounted costs $(£)$ and effects per average women by screening strategy

\begin{tabular}{|c|c|c|c|c|c|}
\hline \multirow[b]{2}{*}{ Screening strategy } & \multicolumn{2}{|c|}{ Difference compared with conventional cytology* } & \multicolumn{3}{|c|}{ Incremental comparison† } \\
\hline & $\begin{array}{l}\text { Life years gained } \\
\text { (discounted) }\end{array}$ & Lifetime cost (discounted) & $\begin{array}{l}\text { Life years gained } \\
\text { (discounted) }\end{array}$ & Lifetime cost (discounted) & Incremental cost effectiveness ratio \\
\hline $\mathrm{A}$ & 0.0019 & 9.9 & - & - & - \\
\hline $\bar{D}$ & 0.0034 & 12.7 & 0.0034 & 12.7 & 3735 \\
\hline $\bar{C}$ & 0.0039 & 20.2 & - & - & - \\
\hline$\underline{E}$ & 0.0049 & 19.1 & 0.0015 & 6.4 & 4233 \\
\hline $\bar{B}$ & 0.0050 & 19.9 & 0.0001 & 0.8 & $£ 18605$ \\
\hline
\end{tabular}

${ }^{*}$ Conventional cytology was least effective strategy.

toptions are compared with next strategy, which was less effective on cost effectiveness frontier

¥0ptions that were extended dominated (that is, inside the cost effectiveness frontier) were excluded.

$\S$ Compared with conventional cytology 


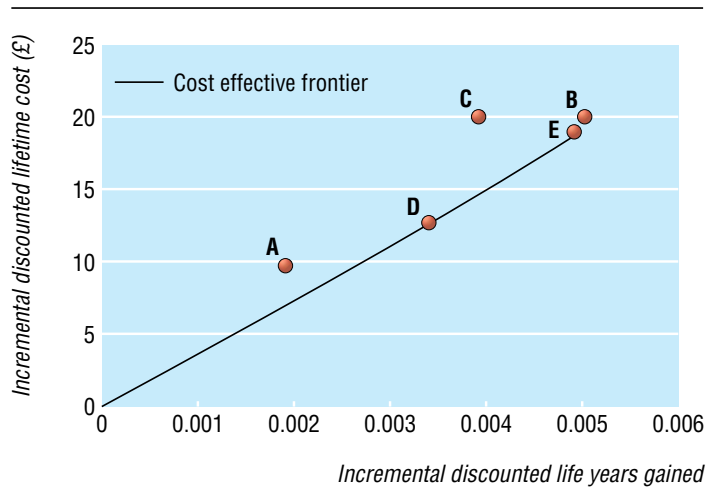

Fig 2 Incremental discounted lifetime costs and effects of alternative screening strategies for cervical cancer compared with screening using conventional cytology only

but are also more expensive. Strategy $\mathrm{C}$ is inside the cost effectiveness frontier and is likely to be dominated (fig 2). These results suggest that strategy $\mathrm{E}$ (no human papillomavirus testing in surveillance) may save slightly less life years but is also slightly less expensive than strategy $\mathbf{B}$ (combined human papillomavirus testing and cytology in surveillance).

\section{Lifetime resource use and invasive cancer mortality}

Table 3 reports estimated lifetime use of resources and mortality from invasive cancer. Compared with screening using only liquid based cytology, there is a $52 \%-86 \%$ reduction in the number of surveillance smears required with the four strategies using human papillomavirus testing. With such strategies, however, the average number of lifetime colposcopies is increased by between $64 \%$ and $138 \%$. This increase in referral for colposcopy is lowest when only women aged more than 35 are tested for human pap- illomavirus. A clear trade-off exists between reduced repeat surveillance smears and increased referral for colposcopy.

The baseline model predicts a $0.49 \%$ lifetime risk of death with liquid based cytology, which compares with current UK data of a $0.56 \%$ lifetime risk of death from invasive cancer. ${ }^{7}$ The model predicts that for women with a borderline result from a routine smear test, $67 \%$ aged less than 35 and $50 \%$ aged 35 or more would test positive for human papillomavirus, and that for women with a mild test result, $81 \%$ aged less than 35 and $67 \%$ aged 35 or more would test positive. These data are similar to those in the findings of the pilot sites. ${ }^{4}$

\section{Sensitivity analyses}

In the one way sensitivity analysis the ranking of the strategies remained similar. The costs associated with liquid based cytology, human papillomavirus testing, and colposcopy had a significant influence on the overall costs. Key areas of uncertainty in the model were the extent preinvasive cancer developed to high grade disease and the sensitivity of cytology for detecting underlying CIN-2 or CIN-3. When the sensitivity of cytology is increased, human papillomavirus testing seems to be less effective, particularly for surveillance of women with initial negative results for human papillomavirus (see bmj.com).

Figure 3 illustrates the results of the probabilistic analysis (strategy $\mathrm{C}$ was excluded as it was dominated, strategy B is not shown but almost overlaps strategy E) and shows that with the current level of evidence there is considerable uncertainty in both the incremental costs and incremental gains that would accrue from using human papillomavirus testing as a triage for women with borderline or mildly dyskaryotic smear results. A negative correlation also exists in the human papillomavirus triage strategies between costs and effects. Finally, the cost effectiveness acceptability frontier shows that if a decision maker is

Table 3 Estimates of average lifetime resource use, risk of treatment for invasive cancer, and mortality

\begin{tabular}{|c|c|c|c|c|c|}
\hline \multirow{2}{*}{ Lifetime resource use } & \multicolumn{5}{|c|}{ Strategy } \\
\hline & A & B & C & D & $\mathrm{E}$ \\
\hline Average No of routine smears & 9.4 & 9.4 & 9.4 & 9.4 & 9.4 \\
\hline Average No of smears in surveillance & 0.77 & 0.11 & 0.24 & 0.37 & 0.30 \\
\hline $\begin{array}{l}\text { Average No of human papillomavirus tests after initial routine } \\
\text { smear }\end{array}$ & 0.00 & 0.42 & 0.43 & 0.25 & 0.40 \\
\hline Average No of human papillomavirus tests in surveillance & 0.00 & 0.11 & 0.21 & 0.08 & 0.00 \\
\hline Average No of colposcopies & 0.21 & 0.41 & 0.40 & 0.31 & 0.35 \\
\hline Average lifetime probability of treatment for invasive cancer & 0.02 & 0.017 & 0.013 & 0.013 & 0.013 \\
\hline Lifetime risk of death from invasive cancer & 0.0049 & 0.0045 & 0.0046 & 0.0046 & 0.0045 \\
\hline
\end{tabular}

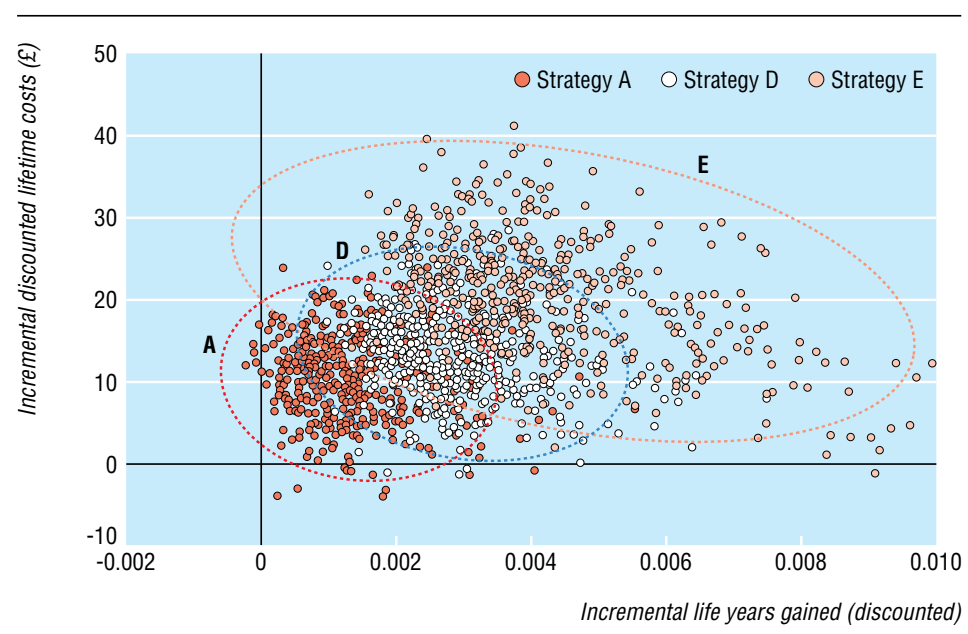

Fig 3 Results of probabilistic sensitivity analysis. Ellipses show 95\% confidence interval for each strategy 
willing to pay between $£ 7500$ (€11 100; \$13 000) and $£ 30000$ per life year gained, strategies when human papillomavirus testing is added to liquid based cytology screening to prioritise all women with borderline or mild dyskaryotic smear results for immediate colposcopy give the greatest net health benefit (see figure on bmj.com).

\section{Discussion}

Using human papillomavirus testing to triage women with mild or borderline cervical smear results is more expensive than repeat cytology but saves slightly more lives. This gain in life expectancy is related both to referring women earlier to colposcopy and to minimising loss to follow up after the initial smear result. This is consistent with the baseline results from a UK study. ${ }^{24}$ Our results can also be compared with a US study, which showed that human papillomavirus testing to triage women with borderline smears saved slightly more lives but was not as expensive as the other screening strategies. ${ }^{25}$

Our study provides further analysis of the alternative strategies for adding human papillomavirus testing. It suggests that the most likely strategy to be cost effective (if society is willing to pay between $£ 7500$ and $£ 30000$ per life year) uses human papillomavirus testing to triage all women with an initial borderline and mild smear result, using cytology to follow-up women only with a negative test result for human papillomavirus.

As with these previous models we used a Markov modelling approach. In this paper we also explored the uncertainty in the model's variables using a probabilistic sensitivity analysis. Alternative modelling approaches exist such as discrete event simulation using individual patient transitions - the advantage of moving to this more complex framework given the lack of availability of patient level data on the natural history is unclear. ${ }^{26}$

The predicted substantial increase in lifetime referral for colposcopy with human papillomavirus testing is of concern (table 3). This finding is confirmed by the observed 12 month outcomes in the pilot study and by the other cost effectiveness model for the United Kingdom. ${ }^{24}$ Nevertheless our conclusions on the cost effectiveness of human papillomavirus testing are robust in the sensitivity analyses when the estimates for cost and effectiveness of colposcopy are varied. Although we have explored a range of screening strategies, further potential options exist such as referring all women with mild cytology results directly to colposcopy or primary human papillomavirus testing.

Finally, this study is limited by the lack of data on the quality of life implications and societal costs (for example, cost of women's time attending for surveillance smears and colposcopies) of using human papillomavirus testing to triage women compared with repeat testing. A trade-off exists between the predicted potential gains in life expectancy and reduction in surveillance smears (52\%-86\%) using human papillomavirus testing, and the negative implications for women of increased lifetime colposcopies (64\%-138\%). This deserves careful consideration and further research.

We thank those involved in the pilots, including the patients, and the laboratory and primary care staff at the pilot sites who assisted in the collection of workload and cost data; staff at the NHS Purchasing and Supplies Agency for providing indicative market prices of consumables and equipment; and the members of the steering and advisory groups. The views expressed are those of the authors and not necessarily those of the Department of Health.

Contributors: RL collected the unit cost data, developed the cost effectiveness model, analysed the data, and wrote the manuscript. AG supervised the collection of cost data and development of the cost effectiveness model and

\section{What is already known on this topic}

Previous models indicate that testing for human papillomavirus to triage women with borderline smear results saves slightly more lives than repeat testing

However, conclusions on the cost implications differ between studies

\section{What this study adds}

Testing for human papillomavirus in women with low grade cytological abnormalities is likely to be cost effective under current UK screening protocols

New cost, epidemiological, and clinical data collected during a large pilot study were used to estimate cost effectiveness

A trade-off exists between the predicted benefits of a small potential gain in life expectancy and reduced surveillance smears from human papillomavirus testing, and the negative implications of an increase in lifetime referrals for colposcopy

critically revised the manuscript. He is guarantor. JW supervised development of the cost effectiveness model and critically revised the manuscript. SM was the principal investigator for the whole evaluation; she assisted with development of cost collection tools and modelling and critically reviewed the paper.

Funding: This study forms part of the independent evaluation of the liquid based cytology/human papillomavirus cervical screening pilot studies commissioned by the policy research programme, Department of Health. Competing interests: None declared.

Ethical approval: Not required.

1 Walboomers JMM, Jacobs MV, Manos MM, Bosch FX, Kummer JA, Shah KV, et al. Human papillomavirus is a necessary cause of invasive cervical cancer worldwide. $J$ Pathol 1999;189:12-9

2 Wright TC, Cox JT, Massad LS, Twiggs LB, Wilkinson EJ. 2001 Consensus guidelines for the management of women with cervical cytological abnormalities. JAMA 2004;287:2120-9.

3 Agence Nationale d'Accreditation et d'Evaluation en Sante. Management of a patient with an abnormal smear. Service recommendations et references professionelles et service evaluation economique. Paris, France: ANAES, 2002:1-92.

4 LBC/HPV Cervical Screening Pilot Studies Group. HPV triage of low grade cytological abnormality: NHS pilot studies.[REF BMJ/2005/295824 in press] [Author: Status of this paper seems to be rejection with offer for resubmission. Please provide an
alternative reference if possible. We give details when papers have 'in press'statusalternative reference if possible. We give details when papers have in press' status-
Response: this is the companion paper and ideally should be published at the same time, it is currently with the co-ordinating editor]

5 Myers ER, McCrory DC, Nanda K, Bastian L, Matchar DB. Mathematical model for the natural history of human papillomavirus infection and cervical carcinogenesis. Am J Epidemiol 2000;151(12):1158-71.

6 West Midlands Cancer Intelligence Unit. Invasive cervical cancer relative survival by stage in the West Midlands: tumours diagnosed 1995-1997 followed up to the end of 2002. University of Birmingham: WMCIU, 2003.

7 Quinn M, Babb P, Brock A, Kirby L, Jones J. Cancer trends in England and Wales 1950-1999. London: Stationery Office, 2001.

8 Government Actuary's Department. Life tables: females England and Wales. London: GAD, 2003

9 Great Britain HM Treasury. Green book, appraisal and evaluation in central government. London: Stationery Office, 2003.

10 Department of Health. Cervical screening programme. England: 2002-2003. Statistical Bulletin 2003/24. London: DoH, 2003.

11 Abryn M, Buntinx F, Van Ranst M, Paraskevaidas E, Martin-Hirsh P, Dillner J. Virologic versus cytologic triage of women with equivocal pap smears: a meta-analysis of the accuracy to detect high grade intraepithelial neoplasia. J Natl Cancer Inst 2004;96:28093.

12 Payne N, Chilcott J, McGoogan E. Liquid-based cytology in cervical screening: a rapid and systematic review. Health Technol Assess 2000;4:1-73.

13 National Audit Office. The performance of the NHS cervical screening programme. London: Stationery Office, 1998.

14 Wolstenholme JL, Whynes DK. Stage-specific treatment costs for cervical cancer in the Wolstenholme JL, Whynes DK. Stage-specific trea
United Kingdom. Eur J Cancer 1998;34:1889-93.

15 Dickson RJ. Management of carcinoma in the cervix. Practitioner 1980;22:899-904

16 Cuzick J, Szareweski A, Terry G, Ho L, Hanby A, Maddox P, et al. Human papillomavirus testing in primary cervical screening. Lancet 1995;345:1533-6. 
17 Noorani HZ, Brown A, Skidmore B, Stuart GCE. Liquid-based cytology and human papillomavirus testing in cervical cancer screening. Technology report No 40. Ottawa, ON: Canadian Coordinating Office for Health Technology Assessment, 2004.

18 Netten A, Rees T, Harrison G. Unit costs of health and social care. Canterbury: University of Kent, 2001.

19 Nanda K, McCrory DC, Myers ER, Bastian LA, Hasselblad V, Hickey JD, et al. Accuracy of the Papanicolaou test in screening for and follow-up of cervical cytologic abnormalities: a systematic review. Ann Intern Med 2000;132:810-9.

20 Briggs AH, Goeree R, Blackhouse G, O'Brien BJ. Probalistic analysis of cost effectiveness models: choosing between treatment strategies for gastroesophagael reflux disease. Med Decis Making 2002;22:290-308.

21 Fenwick E, Claxton K, Sculpher M. Representing uncertainty: the role of cost effectiveness acceptability curves. Health Econ 2001;10:779-87.

22 Cuzick J, Szarewki A, Cubie H, Kitchener H, Luesley D, McGoogan E, et al. Management of women who test positive for high-risk types of human papillomavirus: the HART study. Lancet 2003;362:1871-6.

23 Peto J, Gilham C, Fletcher O, Matthews F. The cervical cancer epidemic that screening has prevented in the UK. Lancet 2004;364:249-56.

24 Kim JJ, Wright TC, Goldie SJ. Cost-effectiveness of alternative triage strategies for atypical squamous cells of undetermined significance. JAMA 2002;287:2382-90.
25 Sherlaw-Johnson C, Phillips Z. An evaluation of liquid-based cytology and human papillomavirus testing within the UK cervical cancer screening programme. Brit J Cancer 2004;91:84-91.

26 Karnon J. Alternative decision modelling techniques for the evaluation of health care technologies: Markov processes versus discrete event simulation. Health Econ 12:837-48.

(Accepted 31 October 2005)

doi $10.1136 /$ bmj.38698.458866.7C

Health Economics Research Centre, Department of Public Health, University of Oxford, Oxford OX3 7LF

Rosa Legood senior researcher

Alastair Gray professor of health economics

Jane Wolstenholme senior researcher

Cancer Screening Evaluation Unit, Institute of Cancer Research, Sutton, Surrey SM2 $5 \mathrm{NG}$

Sue Moss associate director

Correspondence to: R Legood rosa.legood@dphpc.ox.ac.uk 